\title{
Reconstruction of an Electron Energy Distribution Function Using Integrated Data Analysis
}

\author{
Dirk Dodt ${ }^{1}$, Andreas Dinklage ${ }^{1}$, Rainer Fischer ${ }^{2}$, Klaus \\ Bartschat $^{3}$, Oleg Zatsarinny ${ }^{3}$, Detlef Loffhagen ${ }^{4}$ \\ ${ }^{1}$ Max-Planck-Institut für Plasmaphysik, EURATOM Association, \\ Wendelsteinstraße 1, 17491 Greifswald, Germany \\ ${ }^{2}$ Max-Planck-Institut für Plasmaphysik, EURATOM Association, \\ Boltzmannstraße 2, 85748 Garching, Germany \\ ${ }^{3}$ Department of Physics and Astronomy, Drake University, Des Moines, IA 50311, \\ USA \\ ${ }^{4}$ INP Greifswald, Felix-Hausdorff-Str. 2, 17489 Greifswald, Germany \\ E-mail: dirk.dodt@ipp.mpg.de
}

\begin{abstract}
The non-Maxwellian electron energy distribution function (EEDF) in the positive column of a neon dc-discharge was reconstructed from the visible emission spectrum obtained with an overview spectrometer. The analysis is based on Bayesian probability theory (integrated data analysis), which allows for the use of the full information in the spectral data by incorporating all important underlying physical mechanisms. The data are described by a collisional-radiative model and a statistical description of the spectroscopic measurement. An extensive and consistent set of electron impact excitation cross sections and Einstein coefficients obtained through semi-relativistic $B$-Spline $R$-Matrix calculations is employed, and estimates for uncertainties are included in the analysis. The results are consistent with theoretical modeling and reference measurements.
\end{abstract}




\section{Introduction}

Low-temperature plasmas are widely used, e.g., in industrial processes and for lighting purposes [1]. The control and optimization of these plasmas requires diagnostic methods to assess characteristical plasma parameters, among which the electron energy distribution function (EEDF) is a very important one. In this paper a spectroscopic method to infer the EEDF is presented, which makes use of a probabilistic data analysis called Integrated Data Analysis (IDA).

IDA $[2,3]$ is an approach to employ all physical information for inference. A full forward model of the experimental data and a thorough error analysis are used in a probabilistic (Bayesian) calculus to determine quantities of interest along with their uncertainties. The approach enables the analysis of data in cases with complicated dependencies (e.g., mass spectrometry [4]) that are barely analyzable with classical methods. Further benefits are resolution enhancements or a clear increase of significance [5].

The experimental determination of EEDFs is usually performed by measurements of the current-voltage characteristics of a probe in contact with the plasma. The noninvasive spectroscopic approach offers an alternative to these measurements. Probe measurements suffer from the formation of sheaths in the plasma, which limit the applicability of probes in the region of gradients in the plasma parameters. The spectroscopic approach can attain spatial resolution by using a suitable optic and is able to cope with gradients in the parameters of interest. Consequently, the idea to use emission spectroscopy for the determination of the EEDF was brought up long ago [6]. First attempts to use this approach are based on line-ratio techniques mapping the intensities of different spectral lines onto temperatures [7].

Ideally, the line-ratio technique requires some monotonic relation between the desired parameters of the EEDF and the used line-ratios. This is not necessarily fulfilled for all plasmas. The approach used here is based on a method described by Fischer and Dose [8], where a collisional-radiative model (CRM) allows us to relate the line intensities to the EEDF. In the present work, the data descriptive model was extended by direct modeling of the full spectrum, rather than the analysis of derived line intensities. The use of the full spectrum makes it possible to employ sophisticated parameterizations of the EEDF, and hence the approach is not limited to the reconstruction of a small number of parameters in the way line-ratio techniques are. The influence of the atomic data used for the collisional-radiative modeling was addressed. The quantification of the uncertainties of these model parameters are a crucial part of the analysis. The EEDF obtained using the IDA approach was reconstructed together with its uncertainty band, which is a result of the consistent propagation of the uncertainty of the input parameters and the measurement.

The spectroscopic data to be analyzed were obtained using a cylindrical neon dc-discharge. The discharge is a well-examined physical system $([9,10]$ and references therein) with a high reproducibility, thereby allowing for the comparison of equivalent 
discharges with the same geometrical parameters, gas pressure, and electrical circuit. This was taken advantage of to validate the result of the analysis.

The essential prerequisite for the application of the spectroscopic approach is the availability of a collisional radiative model and particularly the atomic data for the spectral lines which are taken into account. The applicability of a similar analysis was already shown for helium discharges [8]. Generally the atomic data basis for the noble gases is sufficient to allow a good collisional-radiative modeling. Lithium beam diagnostics used, e.g., in the edge plasma of high-temperature plasma experiments $[11,12]$ are another example of the application of collisional-radiative modelling for the analysis of spectroscopic data.

The line radiation emitted by excited neutrals in a plasma carries information about the kinetics of the plasma components. In the case of low-temperature plasmas, the main excitation channel is electron-impact excitation, and thus the emission spectrum carries information about the electrons in the plasma. The basic physics of a low-temperature plasma in the parameter regime under consideration can be summarized as follows: The electrons gain energy in the electric field used to sustain the plasma, while the ions absorb only a negligible fraction of the energy because of their much lower mobility. The electrons interact with the neutral gas atoms and ions via various inelastic processes. The energy distribution of the electrons is determined by the balance between heating and inelastic processes. Accordingly, the form of the EEDF generally deviates from a Maxwellian distribution associated with a thermalized electron ensemble.

This paper describes the analysis of the spectral data. After a description of the experimental setup, the analysis procedure and the data descriptive model are presented. The error assessment of the data as well as the proper treatment of uncertainties of the atomic input data are discussed. Different aspects of the analysis can also be found in recent conference proceedings [13].

\section{Experimental Setup}

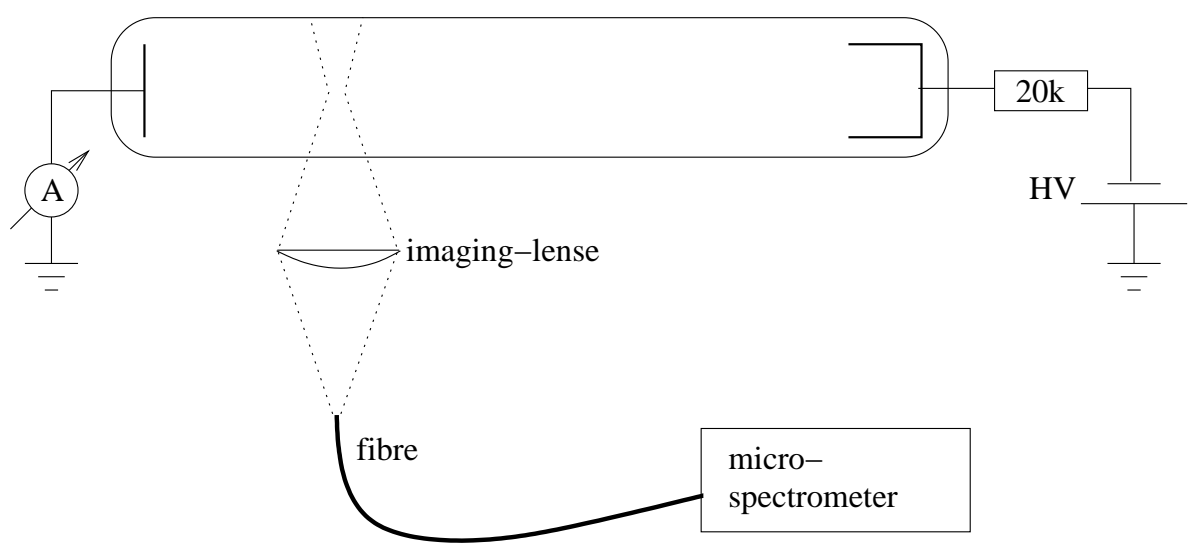

Figure 1. Experimental setup of the spectroscopic measurement. 
The experimental setup shown in fig. 1 is simple and inexpensive. It consists of a dc glow-discharge and a miniature fiber optics spectrometer observing the emitted light. The cylindrical discharge (radius $1.5 \mathrm{~cm}$ ) was operated with neon at a pressure of $67 \mathrm{~Pa}$ and a typical discharge current of $10 \mathrm{~mA}$. The light from the positive column of the discharge was imaged onto an optical fiber, which transmitted the light from the image plane to the spectrometer. The employed imaging-lens had a focal length of $15 \mathrm{~cm}$, resulting in an opening angle of the cone of the line of sight that is much smaller than the depicted one. A Czerny-Turner type spectrometer with a spectral resolution of $\Delta \lambda / \lambda \approx 10^{-3}$, which can be read out digitally, was used to obtain the spectral data. The response of the spectrometer as a function of wavelength was calibrated by replacing the discharge tube with a standard light source in an Ulbricht sphere. It will be shown that this optical setup is sufficient to extract information on the EEDF using the presented analysis procedure.

\section{Integrated Data Analysis of the Spectroscopic Data}

IDA is a formalized way of analyzing experimental data which is based on a probabilistic model for the data (see [5] and references therein). The forward data model defines a mapping from the parameters of interest (and possibly more parameters) to a simulation of the measurement. Typically, Monte-Carlo sampling techniques are used to invert this mapping and to find the parameters of interest compatible with the measured data. The statistical uncertainty of the measurement is taken into account and the resulting uncertainty of the extracted parameters of interest is obtained. A probabilistic description in the framework of Bayesian probability theory allows for taking into account systematic uncertainties of the data model. Beyond the scope of the analysis under consideration, IDA enables a joint analysis of data from different experiments measuring the same or related quantities. Some aspects of IDA will be discussed to provide insight into the data analysis procedure used to extract the EEDF from the spectral data.

The Probabilistic Model of the data consists of the forward model of the data and a description of the statistical fluctuations of the measurements. Specifically, the probability distribution function (pdf) of each element of the data vector $\vec{D}$ needs to be formulated. (Here $\vec{D}$ is given by the measured intensities of each pixel of the CCD chip of the spectrometer.) The joint pdf $P(\vec{D} \mid \vec{\Theta})$ of all elements of $\vec{D}$ is called likelihood. Bayes' Theorem is used to compute the pdf of the model parameters $\vec{\Theta}$ from the likelihood:

$$
P(\vec{\Theta} \mid \vec{D})=P(\vec{D} \mid \vec{\Theta}) \cdot \frac{P(\vec{\Theta})}{P(\vec{D})}
$$

$P(\vec{\Theta} \mid \vec{D})$ is called posterior. The pdf $P(\vec{\Theta})$, which emerges in a formal way here, is called prior. The notations "posterior" and "prior" refer to the status of the knowledge about $\vec{\Theta}$ "with" and "without" (or "after" and "before") taking into account the information contained in the data, which is incorporated in the likelihood. 
The evidence $P(\vec{D})$ is not a function of $\vec{\Theta}$ and therefore does not affect the inference of $\vec{\Theta}$ for a given model. In principle, $P(\vec{D})$ can be obtained from the normalization of the posterior pdf and allows for comparison of different models [14]. Note that $P(\vec{D})$ is not considered in this analysis, because the Metropolis Hastings Monte-Carlo algorithm [15] which was used (see also below) cannot be used to obtain the normalization of $P(\vec{\Theta} \mid \vec{D})$.

The posterior pdf quantifies all information about $\vec{\Theta}$ that can be gained from the data analysis. Generally estimators for the parameters of interest (e.g., the expectation value and rms-variance) are derived from the $P(\vec{\Theta} \mid \vec{D})$. A posterior pdf can be combined with other information from independent sources by using it as prior together with the likelihood of the independent measurement. The computation of $P(\vec{\Theta} \mid \vec{D})$ for each parameter vector involves the computation of the forward model for this set of parameters. The characterization of the high-dimensional posterior with a limited number of computations of the forward model is accomplished using Monte-Carlo sampling.

Implementation of IDA. The practical implementation of a data analysis typically consists of the following steps:

1. Formulation of the forward model for the data

2. Statistical data model: formulation of the likelihood

3. Quantification of prior knowledge including systematic effects

4. Inference: Inversion of the model using Monte-Carlo sampling

5. Focusing: marginalization onto the quantities of interest

The reconstruction of the EEDF, which will be shown in the next section, is based on these steps.

\subsection{Forward Calculation of the Spectroscopic Data}

The forward calculation is a simulation $\vec{D}_{\text {sim }}(\vec{\Theta})$ of the experimental data for a given set of model parameters $\vec{\Theta}$. The forward model is not of probabilistic nature. It is combined with the errors statistics of the measurement to obtain the likelihood, as described below. In order to take advantage of the full information content of the data, the forward model being presented describes the raw data, i.e., the intensities of the different spectrometer pixels, rather than deduced quantities such as line intensities.

Essentially $\vec{D}_{\operatorname{sim}}(\vec{\Theta})$ consists of a stationary collisional-radiative model (CRM) revealing the population densities $n_{i}$ of excited states and ions in the discharge plasma and a description of the spectroscopic measurement. The chain of the different elements of the forward calculation can be summarized as follows:

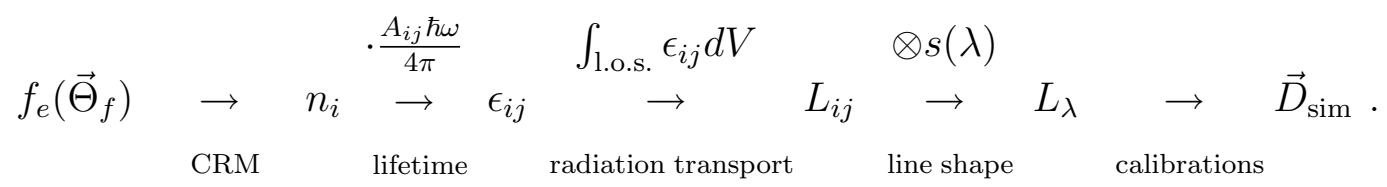


The EEDF $f_{e}$, which depends on the subset $\vec{\Theta}_{f}$ of the model parameters, enters the CRM. The calculated population densities $n_{i}$ are multiplied by the inverse lifetime of the excited states $A_{i j}$ (Einstein coefficient) times the photon energy $\hbar \omega$ and the inverse of the full solid angle $(4 \pi)^{-1}$ to obtain the locally emitted power $\epsilon_{i j}\left[\mathrm{~W} /\left(\mathrm{m}^{3} \cdot \mathrm{sr}\right)\right]$. The radiation has to pass through the plasma before it leaves the discharge device. The apparent lifetime of the excited states is affected by the transport of photons if the absorber density is high, e.g., for transitions to the ground state of the atom [16]. Together with the integration along the line of sight (l.o.s.) of the spectrometer, the description of this opacity results in the effective radiance $L_{i j}$ of each transition.

The line intensities given by the effective radiance have to be convoluted $(\otimes)$ with the line shape $s(\lambda)$ to obtain the effective spectral radiance $L_{\lambda}$. The line shape is determined by the apparatus function, which was obtained here from distinct lines in the spectrum as described in the subsection 3.1.6.

The modeling of the spectrometer output furthermore comprises the description of the response per incident power of each pixel (intensity calibration) and the mapping of pixel numbers onto wavelengths (wavelength calibration). The absolute intensity calibration was measured using a standard light source.

Notation of the Excited States. A convenient labelling of the excited states similar to Paschen's notation is used throughout the paper: The principal quantum number $n$ and the orbital angular momentum $l$ of the excited electron are combined with an energy ordered index, which is one for the state with highest energy among the states with identical $n$ and $l$.

In the context of the CRM, an index is used for the atomic states taken into account. In the present work, we chose 0 for the ground state, 1 to 30 for the excited states (increasing with energy), and 31 for the ionized atom.

3.1.1. Collisional Radiative Model. The population densities of the atomic states $n_{i}$ were described by a set of balance equations accounting for all elementary processes populating or de-populating an atomic level $i$ (equation 3). Ions were treated as an additional state. With a few exceptions, the rates of the elementary processes are proportional to the population density of another excited state. his was used for the solution of the following system of coupled equations for the stationary case:

$$
\begin{aligned}
0 \stackrel{!}{=} \frac{\mathrm{d} n_{i}}{\mathrm{~d} t}= & \underbrace{n_{e}\left[\sum_{k \neq i}\left(\left\langle\sigma_{k i}^{e} v_{e}\right\rangle n_{k}-\left\langle\sigma_{i k}^{e} v_{e}\right\rangle n_{i}\right)\right]}_{\text {Electron (de-)excitation }}+\underbrace{\left[\sum_{k>i} \Lambda_{k i} A_{k i} n_{k}-\sum_{k<i} \Lambda_{i k} A_{i k} n_{i}\right]}_{\text {Radiative transitions }} \ldots \\
+ & \underbrace{n_{a}\left[\sum_{k \neq i}\left\langle\sigma_{k i}^{a} v_{a}\right\rangle n_{k}-\sum_{k \neq i}\left\langle\sigma_{i k}^{a} v_{a}\right\rangle n_{i}\right]}_{\text {Atom collisions }} \ldots
\end{aligned}
$$




$$
\begin{gathered}
-\underbrace{n_{e}\left\langle\sigma_{i \infty}^{e} v_{e}\right\rangle n_{i}}_{\text {Electron impact ionization }}-\underbrace{\left\langle\sigma_{i i}^{a} v_{a}\right\rangle n_{i}^{2}}_{\text {Chemo-ionization }} \\
+\underbrace{n_{e}\left(\beta_{\mathrm{rad}}+\beta_{\mathrm{DE}}\right) n_{\infty}}_{\text {Recombination }}-
\end{gathered}
$$

The rate coefficients of radiative transitions which are not optically thin (see also below) and the rate coefficients for chemo-ionization were calculated by iteratively solving the linearized system of equations.

Electron excitation and de-excitation. The transition rate is given by the rate coefficient $n_{e}\langle\sigma v\rangle=n_{e} \int_{0}^{\infty} \sigma(E) \sqrt{2 E / m_{e}} f_{e}(E) d E$ multiplied by the density of atoms in the initial state of the excitation. Different parameterizations of the energy distribution $f_{e}\left(\vec{\Theta}_{f}\right)$ are employed to determine electron collision rates for the CRM. See also sec. 3.3.2 for a description of the used excitation-cross-sections.

Radiative transitions. For optically thin transitions, the transition rate is given by the Einstein coefficient $A_{k i}$. The escape factor $0<\Lambda_{k i} \leq 1$ accounts for the radiation transport in optically thick regimes. Its computation is described below. See also sec. 3.3.2 for a description of the used coefficients.

Atom collisions. Collisions of the excited atoms with neutral gas atoms lead to excitation transfer between metastable and resonant states of neon, which have the lowest excitation energy among the excited states. The calculation of the rate coefficient is analogous to the electron excitation but much simplified since the atoms are a Maxwellian ensemble at room temperature. The rate coefficient for the respective transitions were taken from [17]

Electron impact ionization. The charge carrier balance is determined by ionization. Only singly charged neon ions are taken into account. The cross-sections were taken from $[18,19]$

Chemo-ionization. The energy of two excited neon atoms is greater than the ionization energy. Therefore collisions between excited atoms may lead to ionization of one of the atoms, while the other atom returns to the ground state. The rate coefficient for the chemo-ionization of two metastable atoms was taken from [20].

Recombination The volume recombination, consisting of the two-body radiative $\beta_{\text {rad }}$ and three-body dielectronic $\beta_{\mathrm{DE}}$ recombination, is listed only for reasons of completeness here. In the considered low pressures regime it can be neglected compared to the recombination at the confining walls of the discharge [21].

Wall de-excitation. Excited atoms or ions coming into contact with the wall of the discharge tube are de-excited to the ground state. The flux $\Gamma_{i}$ was obtained by considering diffusion of the excited atoms and ambipolar diffusion of the ions in the plasma. 

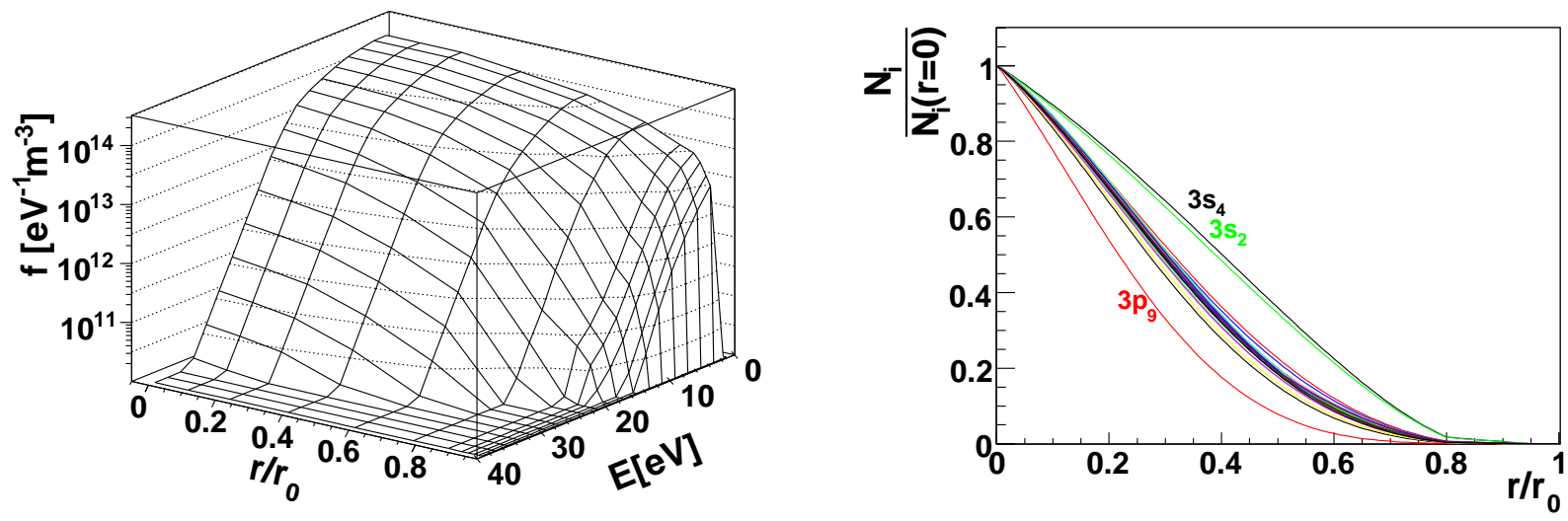

Figure 2. The radial variation of the EEDF as obtained by a hybrid model [9,22] (left) and the radial variation of the 30 excited states considered in the CRM (right, see 3.1 for the notation), obtained with the EEDF depicted on the left; $r_{0}$ is the radius of the discharge tube.The radial variation for most excited states are similar (overlapping curves). Only the three labeled curves, which are the metastable states $3 \mathrm{~s}_{4}$ and $3 \mathrm{~s}_{2}$, as well as one of the $3 p$ states, show a somewhat different behavior.

3.1.2. Radial Dependence of the Elementary Processes and the Population Densities. The discharge device is cylindrically symmetric. In the positive column of the discharge, where the presented measurement was performed, the plasma is homogeneous along the $\mathrm{z}$-axis of the cylindrical tube. Consequently, the plasma in the positive column is to be characterized by a specification of the relevant quantities as a function of the distance $r$ from the discharge center.

The radial dependence of the population densities is relevant for the modeling of the spectroscopic data, because it enters the line of sight integration. In the forward model, the CRM was solved for the rate coefficients at the center of the discharge $(r=0)$, whereas the radial dependence of the population densities was implemented using separate profiles:

$$
n_{i}(r)=n_{i}(r=0) \cdot n_{i}^{r}(r)
$$

Instead of using the often used zeroth order Bessel's function for $n_{i}^{r}(r)$, the profiles were obtained in a preparatory step by solving the CRM at different radial positions. The EEDF at different radial positions was taken from results of hybrid models [9] (see fig. 2). The radial excited state profiles given by [9] could not be used directly, because the $3 p_{i}$ multiplet was implemented as a single combined state in this work. In order to investigate the influence of the assumption of fixed profiles on the line of sight integration, additional scale parameters were introduced for each radial profile (see also table 1).

3.1.3. Radiation Transport. Resonance radiation photons may be re-absorbed by atoms in the final state of the respective transition, particularly for transitions to the ground state. The repetitive emission and absorption resembling many features 
of particle diffusion is called radiation transport. The radiation transport influences the collisional radiative model by changing some of the rates of radiative decays and also affects the amount of light leaving the plasma and reaching the spectrometer. The re-absorption of photons yields an apparent enhancement of the lifetime $\tau_{i}=\left(\sum_{j} A_{i j}\right)^{-1}$ of the excited state, which was quantified using the escape factor $\Lambda_{k i}$ :

$$
A_{k i}^{\prime}=\Lambda_{k i} \cdot A_{k i}, \quad 0<\Lambda_{k i} \leq 1
$$

Approximate models of the radiation transport in discharge tubes were developed in [16]. In these models approximate analytical formulae are used, which take into account the geometrical dimensions of the discharge and the atomic data of the respective transition. The formulae given by [16] were employed in the present model for transitions to the ground state.

3.1.4. Optical Depth of Transitions to Metastable States. The second-highest populated states of the neon atoms are the metastable states $\left[2 \mathrm{~s}^{2} 2 \mathrm{p}^{5}\left({ }^{2} \mathrm{P}_{3 / 2}^{\circ}\right) 3 \mathrm{~s}\right] J=2\left(1 \mathrm{~s}_{5}\right)$ and $\left[2 \mathrm{~s}^{2} 2 \mathrm{p}^{5}\left({ }^{2} \mathrm{P}_{1 / 2}^{\circ}\right) 3 \mathrm{~s}\right] J=0\left(1 \mathrm{~s}_{3}\right)$, which have a negligible radiative decay rate. Their depopulation is caused by electron impact de-excitation, collisions of the metastables with the glass tube, and collisional transfer to resonant states. The density of the metastable atoms is sufficiently high to cause an optical depth of transitions to these states. The formulae for the escape factors to the ground state are not directly applicable here, since the absorber density varies spatially. However, the result of the approximation [16] can be used to obtain lower limits for the actual escape factors by inserting the radial maximum of the respective metastable density. The reason for this estimation is that the enhancement of the lifetime has to be smaller than the enhancement that would result from the maximal density.

In order to account for the variation of the metastable densities as a function of the radius, effective absorber densities were introduced. These were used with the formulae of [16]. The values of the effective densities were fitted to the measured spectra in the inversion procedure.

3.1.5. Line of Sight Integration. The contribution of the transition $j \rightarrow i$ to the overall radiance of the plasma surface has to be obtained by an integration along the line of sight (l.o.s.):

$$
L_{i j}=\int d s \frac{1}{A_{\perp}(s)} \int d A_{\perp} \varepsilon_{i j}\left(s, r_{\perp}\right) .
$$

Here $A_{\perp}(s)$ is the area perpendicular to the l.o.s. of the plasma volume, which is imaged onto the optical fiber. $A_{\perp}(s)$ depends on the position $s$ along the l.o.s. and is computed taking into account the area of the optical fiber, the opening angle of the optics, and the enlargement factor of the imaging optics. Equation (6) can be regarded as the average of $\varepsilon_{i j}$ over the area $A_{\perp}(s)$, which is integrated with respect to $s$. 
3.1.6. Line Shape. The effective spectral radiance $L_{\lambda}(\lambda)$ can be regarded as the intensity distribution of the light after passing the dispersing grating of the spectrometer. It is obtained by convolving the spectral radiance of the plasma $L_{\text {plasma }}(\lambda)$ with the apparatus function $s_{a}$ :

$$
L_{\lambda}(\lambda)=\int L_{\text {plasma }}\left(\lambda^{\prime}\right) s_{a}\left(\lambda-\lambda^{\prime}\right) d \lambda^{\prime}
$$

Since the width of the apparatus function is large compared to the line broadening effects in the plasma, the latter may be neglected and the line shape is given by the apparatus function $s=s_{a}$. In our analysis, the effective spectral radiance was obtained by summing up the radiance of each transition $L_{i j}$ multiplied by the line shape:

$$
L_{\lambda}(\lambda)=\sum_{i j} L_{i j} s\left(\lambda-\lambda_{i j}\right)
$$

where $\lambda_{i j}$ is the wavelength of the light emitted by the respective transition.

As a first approximation, the apparatus function of a spectrometer using a grating as its dispersive element can be described by a Gaussian function (see fig. 3). In order to model the form of the function with higher precision, a spline was fitted to the measured line profiles. For this purpose, well-separated spectral lines at different wavelengths were shifted and rescaled, and a smoothing spline was fitted to all points of the superimposed lines. Emission spectra of Helium and Krypton discharges were used to obtain an adequate number of lines at different wavelengths. Two different spline approximations were used for lines located above and below $\lambda=750 \mathrm{~nm}$, because the data show a change of the line shape for large wavelengths. An error $\sigma_{s}\left(\lambda-\lambda^{\prime}\right)$ was determined from the residuals of the smoothing spline fit. It quantifies the variation of the apparatus function for spectral lines with different intensities and at different wavelengths. (Compare also section 3.3.)
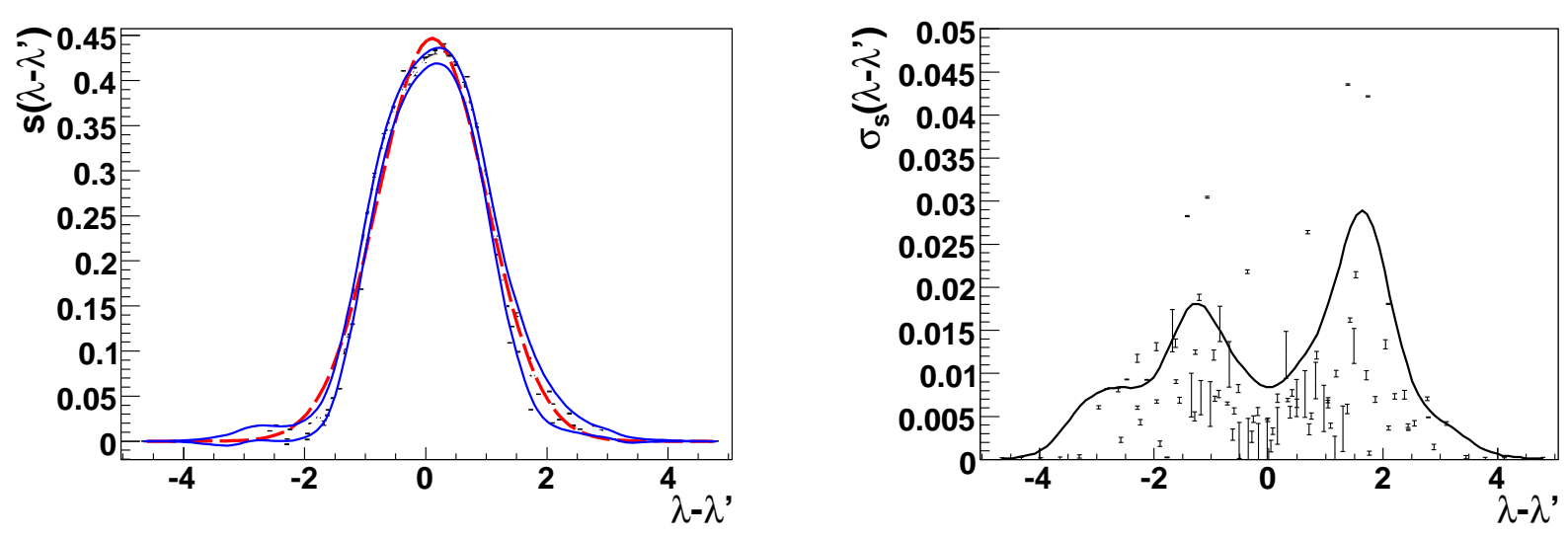

Figure 3. Left: Line profile of the spectrometer obtained by shifting and scaling different lines (see text). The error band of the smoothing spline (solid curves, blue) is shown together with a Gaussian fit (broken curve, red) for comparison. Right: The error of the profile is determined using the absolute value of the residuals from the smoothing spline weighted with their respective statistical errors. 
3.1.7. Calibration of the Spectrometer. The description of the response of the CCD chip has to take into account the dark current $D_{\text {dark, } i}$ and calibration factor $C_{i}$ for each pixel. The mapping of pixel numbers to wavelengths was implemented using a second-order polynomial:

$$
D_{\mathrm{sim}, i}=C_{i} \cdot L_{\lambda}\left(\lambda_{i}\right)+D_{\mathrm{dark}, i} ; \quad \lambda_{i}=\lambda_{0}+\lambda^{\prime} i+\lambda^{\prime \prime} i^{2} .
$$

The output of the used spectrometer $D_{\text {sim }, i}$ for each pixel $i$ has a dynamic range of 12 bit and is expressed in analog-to-digital units (ADU).

The fluctuation of the dark current and the readout noise $\sigma_{\mathrm{ro}, i}$ were determined by repeated measurements without light incidence. The parameters of the wavelength mapping $\lambda_{0}, \lambda^{\prime}, \lambda^{\prime \prime}$ were fitted to the data together with parameters of the EEDF.

The calibration factor was determined using a standard light source. The response of the CCD pixels $s_{i}$, when exposed to the spectral radiance $L_{\mathrm{s}}\left(\lambda_{i}\right)$ of the standard light source, was measured and $C_{i}$ was computed:

$$
C_{i}=\frac{T}{T_{\mathrm{s}}} \frac{\left(s_{i}-D_{\mathrm{dark}, i}\right)}{L_{\mathrm{s}}\left(\lambda_{i}\right)}
$$

Here $T$ and $T_{\mathrm{s}}$ are the exposure times of the spectral measurement of the plasma and the standard light source. As the calibration factor is the result of a measurement, it is also subject to statistical uncertainty with the standard deviation $\sigma_{C, i}$. Assuming independent and Gaussian-distributed noise of the spectral measurement, the uncertainty of $C_{i}$ can be estimated using Gaussian error propagation:

$$
\sigma_{C, i}=\frac{T}{T_{\mathrm{s}} L_{\mathrm{s}}\left(\lambda_{i}\right)} \cdot \sigma_{\mathrm{spec}, i}
$$

Here $\sigma_{\mathrm{spec}, i}$ is the rms-variance of the spectral measurement of the standard light source (see also next section).

\subsection{Formulation of the Likelihood Function}

The likelihood $P(\vec{D} \mid \vec{\Theta})$ states the probability to obtain the measured data $\vec{D}$ given the parameter set $\vec{\Theta}$. It represents the statistical model of the measurement. The likelihood of each pixel $D_{i}$ can be obtained from the value of the model $D_{\operatorname{sim}, i}$ and the distribution of the statistical error of this pixel. For Gaussian-distributed independent statistical fluctuations the likelihood of the data is given by:

$$
P(\vec{D} \mid \vec{\Theta})=\frac{1}{\prod_{i} \sqrt{2 \pi \sigma_{i}^{2}}} \exp \left\{-\frac{1}{2} \sum_{i} \frac{\left(D_{i}-D_{\text {sim }, i}\right)^{2}}{\sigma_{i}^{2}}\right\} .
$$

Here $\sigma_{i}$ is the uncertainty of the measurement of pixel $i$ of the CCD given in ADU. It consists of two contributions: the photon noise $\sigma_{\mathrm{ph}}$ and the readout noise $\sigma_{\mathrm{ro}, i}$. The photon noise is caused by the statistical incidence of photons at the detector. It follows photon statistics and $\sigma_{\mathrm{ph}}$ is proportional to the number of photo-electrons generated in the CCD. The readout noise was assumed to be independent of the signal amplitude and the same for all pixels. 


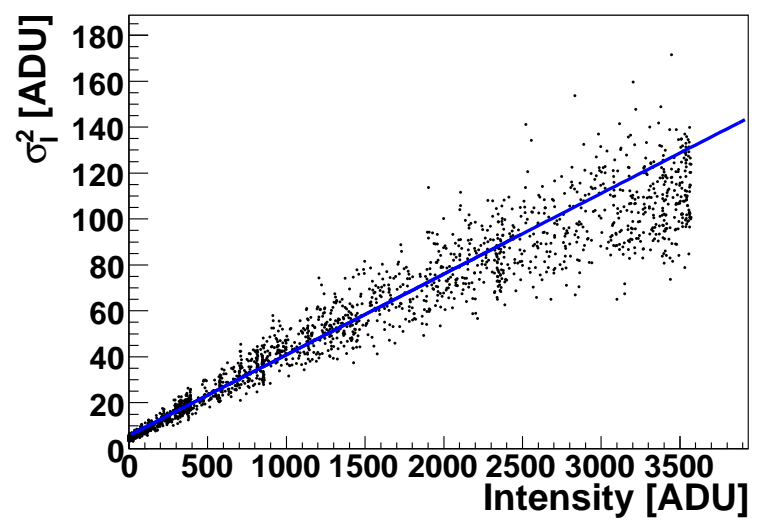

Figure 4. The statistical fluctuation of the spectrometer is obtained by repeated measurements of the spectrum of the standard light source. It is described by counting statistics (Poisson distribution, $\sigma^{2} \propto I$ ). The variance $\sigma_{\mathrm{spec}, i}^{2}$ is obtained from 100 exposures and is shown as a function of the intensity measured in analog-to-digital units. The straight line is fitted to data in the range from 0 to 2500 ADU.

Determination of the Statistical Error of the Spectral Measurement. As described in [23], the noise of the signal obtained by a CCD sensor depends on the amplitude $D_{i}-D_{\text {dark }, i}$ in the following way:

$$
\sigma_{\mathrm{spec}, i}^{2}=E_{\gamma} \cdot\left(D_{i}-D_{\mathrm{dark}, i}\right)+\sigma_{\mathrm{ro}}^{2} .
$$

The conversion factor $E_{\gamma}$ is equal to the inverse number of photon electrons per ADU. Experimentally the dependence of the variance $\sigma_{\text {spec }, i}^{2}$ on the signal amplitude was determined from repeated measurements of the spectrum of the standard light source. The variance of each pixel of the spectrum of the standard light source was plotted against $D_{i}-D_{\text {dark }, i}$ (see fig. 4). The parameters of equation. (13) were fitted to the data in fig. 4 , with the following result:

$$
\begin{aligned}
E_{\gamma} & \approx \frac{1}{28.46} ; \\
\sigma_{\text {ro }}^{2} & \approx 5.72 \mathrm{ADU}^{2} .
\end{aligned}
$$

Effective Width of the Likelihood $\sigma_{\mathrm{eff}, i}$. The uncertainty of the apparatus function $\sigma_{s}\left(\lambda-\lambda^{\prime}\right)$ (see sec. 3.1.6) and of the relative intensity calibration $\sigma_{\mathrm{C}, i}$ (see sec. 3.1.7) were taken into account by using an effective width of the Gaussian likelihood:

$$
\begin{aligned}
P(\vec{D} \mid \vec{\Theta}) & =\frac{1}{\prod_{i} \sqrt{2 \pi \sigma_{i}^{2}}} \exp \left\{-\frac{1}{2} \sum_{i} \frac{\left(D_{i}-D_{\text {sim }, i}\right)^{2}}{\sigma_{\text {eff }, i}^{2}}\right\} . \\
\sigma_{\text {eff }, i}^{2} & =\sigma_{\text {spec }, i}^{2}+\sigma_{\mathrm{C}, i}^{2}+\sum_{j k} L_{j k}^{2} \sigma_{f}^{2}\left(\lambda_{i}-\lambda_{j k}\right) .
\end{aligned}
$$

The usage of the effective likelihood is equivalent to the introduction of additional model parameters with Gaussian priors and subsequent marginalization, as derived in [24]. 


\subsection{Prior Distributions}

For all parameters treated in a probabilistic way, the prior pdfs $P(\vec{\Theta})$ have to be specified. This means that the available knowledge about the respective parameter is quantified without taking into account the experimental data,

Generally speaking, there are two kinds of parameters that have to be described probabilistically: First, the parameters of interest, which are inferred from the data and are to be determined in the inversion procedure. Second, parameters which we are not interested in, but whose values are not known precisely. In table 1 the parameters of the forward model and the assigned priors are summarized. The choice of the prior distributions is discussed in the following paragraphs.

Table 1. Summary of the parameters $\vec{\Theta}$ used in the forward model. Parameters, for which no prior is stated, are not treated probabilistically The Gaussian priors of the line shape and the intensity calibration of each pixel are taken into account by the effective width of the likelihood (see text).

\begin{tabular}{cll}
\hline Symbol & Parameter Description & Remarks, Prior \\
\hline$\vec{\Theta}_{f}$ & Parameters of the EEDF & Flat prior \\
$N_{e}$ & Electron density & Uniform prior \\
$\sigma_{i j}$ & Scale of electron impact excitation cross sections & Gaussian prior \\
$A_{i j}$ & Einstein coefficients & Gaussian prior \\
$D_{m}$ & Diffusion coefficient of metastables & - \\
$D_{I}$ & Ambipolar diffusion coefficient of Ions & - \\
$\Lambda_{i j}$ & Escape factors (EF) of transitions to ground state & - \\
$C_{3 \mathrm{~s} 4 / 3 \mathrm{~s} 2}$ & EF of transitions to the metastable levels & Flat prior for eff. densities \\
$p_{\mathrm{Ne}}$ & Gas pressure & - \\
$T_{\mathrm{Ne}}$ & Gas temperature & - \\
$r$ & Diameter of the discharge tube & - \\
$n_{\mathrm{Duran}}$ & Refractive index of glass & - \\
$d$ & Thickness of glass & - \\
$n_{5 s i, 4 d i}$ & Populations of unmodeled atomic levels & Exponential prior \\
$n_{3 s i, 3 p, 3 d, . .}$ & Radial profiles of the excited state densities & Gaussian prior \\
$s(\lambda)$ & Line shape & Gaussian prior, analytic \\
$\lambda_{0}, \lambda^{\prime}, \lambda^{\prime \prime}$ & Wavelength calibration & Uniform priors \\
$C_{i}$ & Intensity calibration & Gaussian prior, analytic \\
$C_{\text {scale }}$ & Scale of intensity calibration & Gaussian prior \\
\hline
\end{tabular}

3.3.1. Parameters of interest. For the parameters we are interested in no additional information was included in the analysis and flat prior distribution were employed. The parameterization of the EEDF is given in equation (21). Beneath the parameters of the equation (21), which are the electron temperature and the values of the spline nodes, 
also the electron density was extracted from the spectral data and a flat prior was used for $N_{e}$.

3.3.2. Atomic Data. For the electron excitation cross sections and Einstein coefficients, a dataset from semi-relativistic $B$-spline Breit-Pauli $R$-Matrix (BSRM) calculations was used. Details of these calculations can be found in refs. [26,27]. Briefly, they are based on a close-coupling description of e - Ne collisions, including the lowest (in energy) 31 target states. Using term-dependent, and hence non-orthogonal orbital sets, which were individually optimized for each target state of interest, allowed for a highly accurate target description with a relatively small number of configurations in the configurationinteraction expansion. The above data were chosen, since they represent a complete, and internally consistent, dataset for all transitions of interest.

Data for oscillator strengths from the same BSRM model were combined with those from the atomic line database NIST [28]. Where data from NIST are available, a weighted average of the Einstein coefficients was computed using the uncertainties stated by NIST and the ones described below. The cross sections for ionization of neon in the ground and excited states were taken from [18] and [19].

The uncertainties of the electron-impact excitation cross sections were incorporated using a single, energy-independent scale parameter for each cross section. This is a reasonable choice, since in BSRM calculations the energy dependence of the cross sections is known to be more reliable than the absolute scale. [29]. The use of a single scale parameter also allowed for an efficient implementation in the model.

A log-normal distribution [30] was used as prior for the scale parameters. Its pdf is given by:

$$
\begin{aligned}
& G_{\log }(x \mid \tilde{\mu}, \tilde{\sigma})=\frac{e^{-(\ln x-\tilde{\mu})^{2} /\left(2 \tilde{\sigma}^{2}\right)}}{x \tilde{\sigma} \sqrt{2 \pi}} ; \\
& \mu=e^{\tilde{\mu}+\tilde{\sigma}^{2} / 2} ; \quad \sigma_{\mathrm{rms}}=\left(e^{\tilde{\sigma}^{2}}-1\right) e^{2 \tilde{\mu}+\tilde{\sigma}^{2}}
\end{aligned}
$$

with an expectation value $\mu$ and a rms-variance $\sigma_{\text {rms }}$. The relative width $\left(\sigma_{\text {rms }} / \mu\right)$ used is listed in table 2. The numbers were chosen according to details of the BSRM calculations and the available independent experimental validations of the cross sections (see $[26,27,31,32])$.

Table 2. Uncertainties of the excitation cross sections.

\begin{tabular}{lc}
\hline final state & $\begin{array}{c}\sigma_{\text {rms }} / \mu \text { of log-normal distribution } \\
\text { (relative error) }\end{array}$ \\
\hline $2 \mathrm{p}^{5} 3 \mathrm{~s} J=1$ & $10 \%$ \\
$2 \mathrm{p}^{5} 3 \mathrm{~s} J=2$ & $20 \%$ \\
$2 \mathrm{p}^{5} 3 \mathrm{p}$ & $40 \%$ \\
$2 \mathrm{p}^{5} 4 \mathrm{~s}$ & $60 \%$ \\
$2 \mathrm{p}^{5} 3 \mathrm{~d}$ & $60 \%$ \\
\hline
\end{tabular}


The uncertainty of the Einstein coefficients $A_{i j}$ was assessed by considering the results of the BSRM calculations in the length and the velocity form of the electric dipole operator. Since both should, in principle, yield the same result, the difference was used as an estimate for the uncertainty [33]. Figure 5 shows the relative difference between the results in the two forms of the dipole operator as a function of the absolute value of the Einstein coefficient. For small $A_{i j}$, the calculations are expected to be less accurate compared to stronger transitions. Consequently, the relative width of the prior distribution $\sigma_{\text {rms }} / \mu$ in fig. 5 was chosen depending on the value of $A_{i j}$. It is taken as large as the biggest relative difference for a certain value of $A_{i j}$, but never smaller than $10 \%$. Where both numbers were available, a weighted average of the Einstein coefficients from the BSRM calculations and the NIST database [28] was computed.

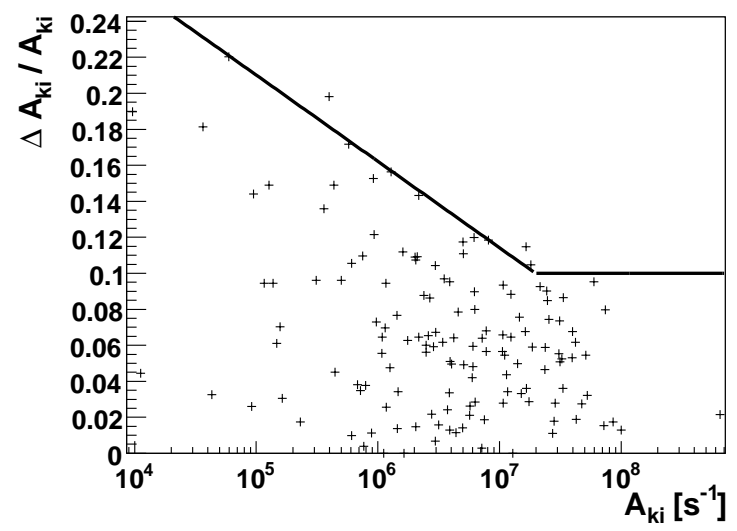

Figure 5. Absolute value of the relative difference $2\left|A_{i j,(v)}-A_{i j,(l)}\right| /\left(A_{i j,(v)}+A_{i j,(l)}\right)$ between the BSRM results for the Einstein coefficients in the length and velocity form of the dipole operator plotted as a function of the absolute value of the Einstein coefficient. The straight line depicts the rms-width of the prior distribution as a function of the absolute value of the coefficient (see text).

3.3.3. Escape Factors to Metastable States. The effective densities of the metastable states $\left(1 \mathrm{~s}_{5}\right.$ and $\left.1 \mathrm{~s}_{3}\right)$ were implemented by means of correction factors $C_{3 \mathrm{~s} 4 / 3 \mathrm{~s} 2}$ to the densities obtained by the collisional-radiative model (cf. 3.1.4). These correction factors have to be smaller than unity, since the maximum of the population density is located in the center of the discharge, for which the CRM was calculated. A uniform prior distribution between $0<C_{3 \mathrm{~s} 4 / 3 \mathrm{~s} 2}<1$ was used for the factors.

3.3.4. Population Densities of Unmodeled Levels. In the spectral range described by the forward model, there are a few lines originating from excited states, for which no cross-section data is available. In order to complete the forward model in these areas, the population densities of these states $\left(4 d_{i}, 5 s_{i}\right)$ were introduced as parameters of the forward model. The population densities are expected to be somewhat lower than the lower-lying states that are described by the CRM. In concordance with the MaxEnt 
principle [34], exponential distributions were used as priors. The expectation value of the respective density was estimated using a Boltzmann factor for an electron temperature of $4 \mathrm{eV}$.

3.3.5. Prior Distributions of the Radial Profile Integrals. An assumption about the radial profiles of the excited states populations is necessary for the line of sight integration. In order to account for the uncertainty of this assumption scale factors were introduced for the radial profiles. A log-normal prior distribution with expectation value of 1 and a rms-variance of 0.1 was used. The assignment of the rms-variance was based on the variation of the integral of the radial profiles for profiles taken directly from [9] compared to the multiplet-resolved computation described above.

3.3.6. Priors of the Wavelength Calibration. The wavelength calibration is well determined by the data. Hence the posterior for $\lambda, \lambda^{\prime}$, and $\lambda^{\prime \prime}$ is strongly dominated by the likelihood. Uniform prior distributions were used for the parameters of the calibration.

3.3.7. Priors of the Absolute Intensity Calibration. In addition to the uncertainty of the calibration factors that is caused by the finite precision of the calibration measurement (cf. 3.1.7), there is an uncertainty in the (wavelength-integrated) radiance of the standard light source. The uncertainty of the calibration measurement of each pixel was incorporated in the effective width of the Gaussian likelihood (equation 3.2). The uncertainty of the radiance, which is specified by the supplier of the standard light source (Labsphere Inc., http: \\www.labsphere.com), was taken into account by a scale parameter for the spectral radiance of the standard light source. A log-normal prior distribution with an expectation value of 1 and a rms-variance of 0.05 was used as prior.

3.3.8. Priors of the Apparatus Function. As described in 3.1.6, the apparatus function extracted from measured spectra is subject to uncertainty. This uncertainty was accounted for by the effective width of the Gaussian likelihood described above. The description with an effective likelihood is equivalent to the introduction of additional parameters with Gaussian prior distributions and a subsequent marginalization [24].

\subsection{Inversion Procedure}

The characterization of the posterior (equation 1), i.e. the numerical computation of estimators for the parameters of interest of the high dimensional pdf, is performed using a Markov chain Monte-Carlo (MCMC) algorithm, (see e.g. [15]).

A Markov chain is a sequence of random variables $\left\{\vec{\Theta}_{1}, \vec{\Theta}_{2}, \ldots\right\}$ where the next state $\vec{\Theta}_{t+1}$ is sampled from a distribution $P\left(\vec{\Theta}_{t+1} \mid \vec{\Theta}_{t}\right)$, called the transition kernel, which only depends on the current state of the chain $\vec{\Theta}_{t}$. A time-homogeneous Markov chain 
whose transition kernel does not depend on $t$, will converge to a unique stationary distribution $\phi$. When an arbitrary starting state $\vec{\Theta}_{0}$ was chosen, the chain will take a number of steps before it converges to the stationary distribution. A Markov chain with a selectable desired stationary distribution, can be constructed e.g. using the MetropolisHastings algorithm. The transition kernel of the Metropolis-Hastings algorithm consists of a proposal distribution from which a sample is drawn and accepted with a certain probability depending on the desired $\phi$ of the chain. The dependece of the algorithm on the previous state arises because $\phi$ is evaluated at $\vec{\Theta}_{t}$ and $\vec{\Theta}_{t+1}$.

In the present case the desired stationary distribution is the posterior pdf (equation 1), whose numerical implementation is based on the likelihood and the priors described above. A Cauchy distribution for each element of the parameter vector $\vec{\Theta}$ is used as proposal distribution (single-component Metropolis-Hastings algorithm [15]). In a so called burn-in phase the width of the proposal distributions is adjusted in a way, that a fraction of roughly 0.35 of the drawn proposal samples are accepted during the Metropolis-Hastings algorithm. As a matter of experience this acceptance rate allows a good convergence of the chain. During the burn-in the chain also departs from the initial state of the chain, thus getting rid of the influence of the initial values of the inversion procedure.

The numerical computations were performed on a linux cluster. On each CPU a chain of the length of $\mathcal{O}\left(10^{4}\right)$ samples was computed. A burn-in of a few hundreds of samples was used in each chain to adjust the width of the proposal distributions. The samples of the burn-in were not used for the characterization of the posterior.

The set of samples $\left\{\vec{\Theta}_{1}, \vec{\Theta}_{2}, \ldots, \vec{\Theta}_{n}\right\}$ obtained this way was used for the estimation of the parameters of interest. The possibility to formulate estimators for quantities derived from the actual model parameters was employed to depict the reconstructed EEDF $f_{E}(\vec{\Theta})$ at a given energy $E$. The expectation value for $f_{E}$ was extracted from the $\mathrm{MC}$ samples in the following way:

$$
\left\langle f_{E}(\vec{\Theta})\right\rangle=\int f_{E}(\vec{\Theta}) P(\vec{\Theta} \mid \vec{D}) d \vec{\Theta} \simeq \frac{1}{n} \sum_{i}^{n} f_{E}\left(\vec{\Theta}_{i}\right)
$$

Its variance is given by:

$\operatorname{Var}\left(f_{E}(\vec{\Theta})\right)=\left\langle f_{E}^{2}(\vec{\Theta})\right\rangle-\left\langle f_{E}(\vec{\Theta})\right\rangle^{2} \simeq \frac{1}{n} \sum_{i}^{n} f_{E}^{2}\left(\vec{\Theta}_{i}\right)-\left(\frac{1}{n} \sum_{i}^{n} f_{E}\left(\vec{\Theta}_{i}\right)\right)^{2}$.

In the plots of the results section, which are showing the reconstructed EEDF, $f_{E}$ is shown as given in equation (21) and multiplied by the electron density $N_{e}$. The parameters of interest that are depicted in the plots are accordingly: $N_{e}, T_{e}$ and the values of the spline nodes of equation (21).

3.4.1. Parameterization of the EEDF. The parameterization used to implement the EEDF in the reconstruction is a Maxwellian distribution multiplied by an energy 

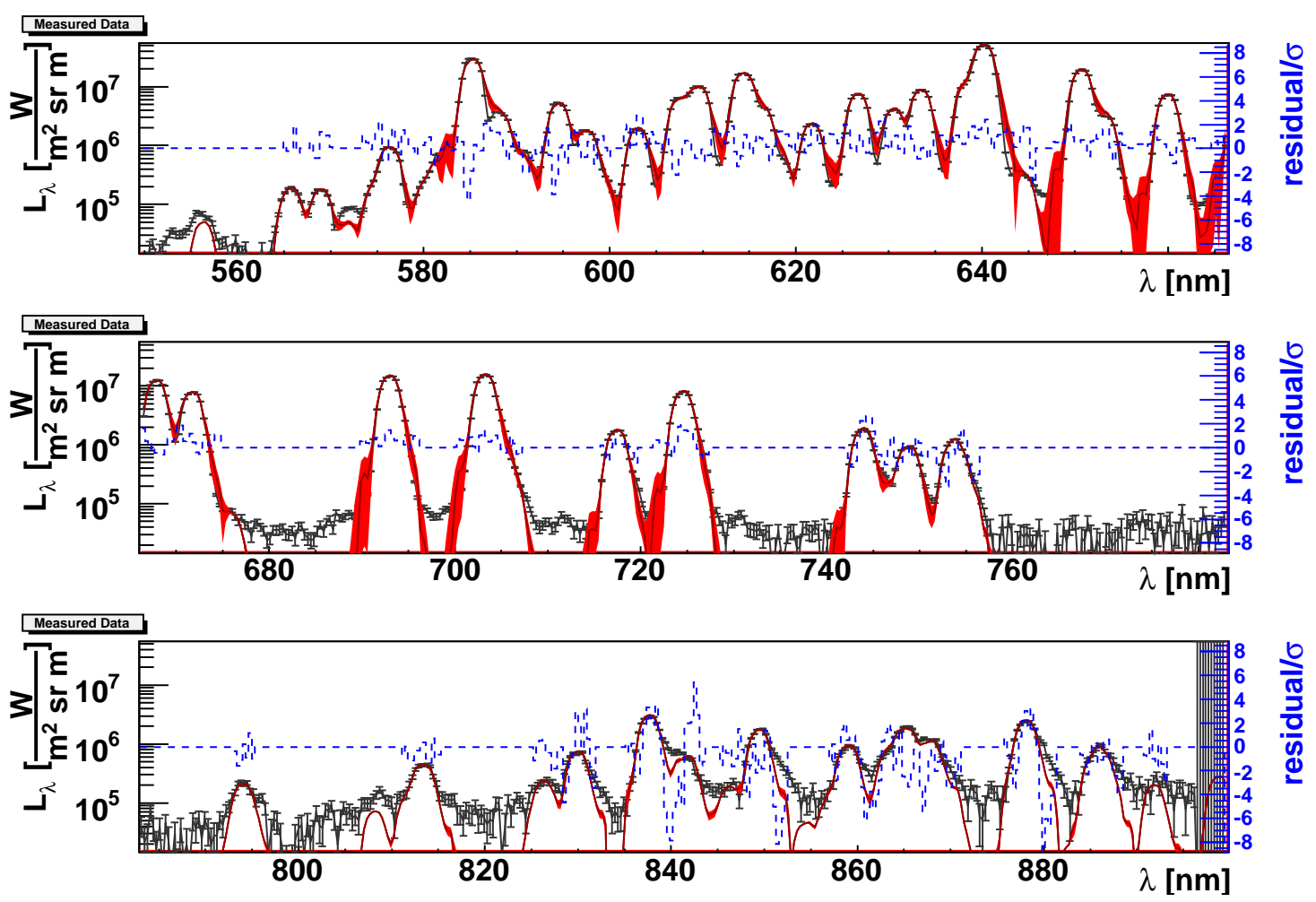

Figure 6. Result of the forward model. The intensity is shown as a function of wavelength on a logarithmic scale. The curve inside the light-colored (red) area depicts the modeled spectrum with the uncertainty of the apparatus function. The black curve with error bars shows the measurement and its uncertainties. The dashed (blue) line represents the difference between model and measurement in units of standard deviations.

dependent factor:

$$
F_{M}(E)=\underbrace{2 \sqrt{\frac{E}{\pi\left(k T_{e}\right)^{3}}} \exp \left\{\frac{-E}{k T_{e}}\right\}}_{\text {Maxwellian }} \times \exp \left\{f_{\text {Spline }}(E)\right\} .
$$

The modification factor allowed us to describe EEDFs that deviate from Maxwellian distributions, as expected from independent kinetic modeling. The factor was implemented using either an approximating spline [35] or a piecewise linear interpolated function in the argument of an exponential function. The exponential in equation (21) is used to assure positivity of the EEDF.

\section{Results and Discussion}

For a successful inversion it is necessary, though not sufficient, that the forward model provides a consistent and unbiased description of the measured data. Figure 6 depicts the result of the forward model together with the measurement. The dashed (blue) curve shows the difference between model and measurement in units of standard deviations. 


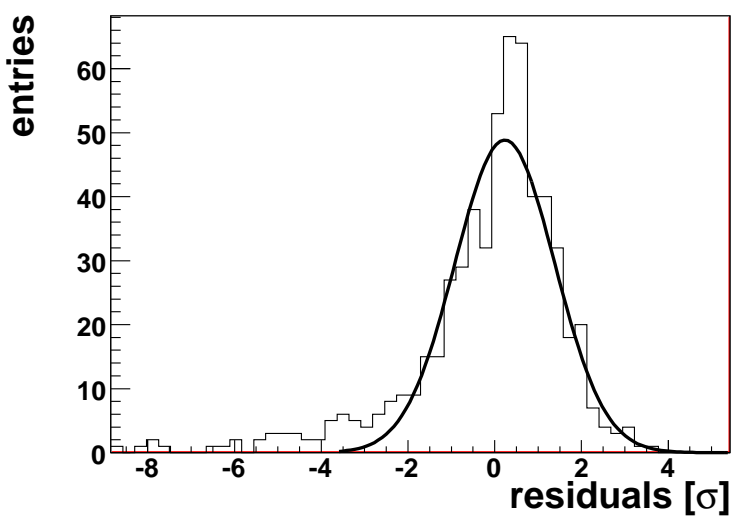

Figure 7. The distribution of the differences between model and measurement is shown in units of standard deviations. The fitted Gaussian curve has a width of 1.14 and a mean of 0.23 . The histogram is the projection of the dashed (blue) curve in fig. 6 .

It can be seen that every feature of the neon spectrum in the considered range of wavelengths is incorporated in the model (note the logarithmic scale). This is possible due to the extensive set of atomic structure and collision data available from the BSRM calculations. The only significant deviations between model and data are located at wavelengths above $800 \mathrm{~nm}$. The residuals are mainly located at the large-wavelength side of some spectral lines. An imperfect description by the apparatus function at large wavelengths is a possible explanation for this behavior. The use of the second apparatus function at high wavelengths (see also 3.1.6) improves but does not solve this discrepancy. This is also indicated by the presence of the tail towards negative residuals in fig. 7, where the overall distribution of the residuals between model and measurements is shown. Despite the mentioned tail, the bulk of the distribution has almost the shape of a Gaussian distribution with width one in units of standard deviations. Keeping in mind, that the modelled data ranges over almost three orders of magnitude, this indication of the consistency of model and data as well as the correct assignment of error statistics can be regarded as an achievement.

\subsection{Reconstruction of Simulated Spectral Data}

The reliability of the inversion procedure was verified by inverting a simulated spectrum obtained using the forward model. The parameters of interest, which were used to generate the simulated data, have to be reobtained if the reconstruction works correctly and the model uncertainties are reflected in the result.

The parameterization given by equation (21), consisting of a Maxwellian distribution and a spline allowing form-free deviations, was used for the EEDF of the simulated data. The correcting spline was chosen such that the EEDF approximates the reference EEDF [9]. The result of the inversion is shown in fig. 8 together with the EEDF used for the generation of the simulated spectrum. The color-coded histograms 


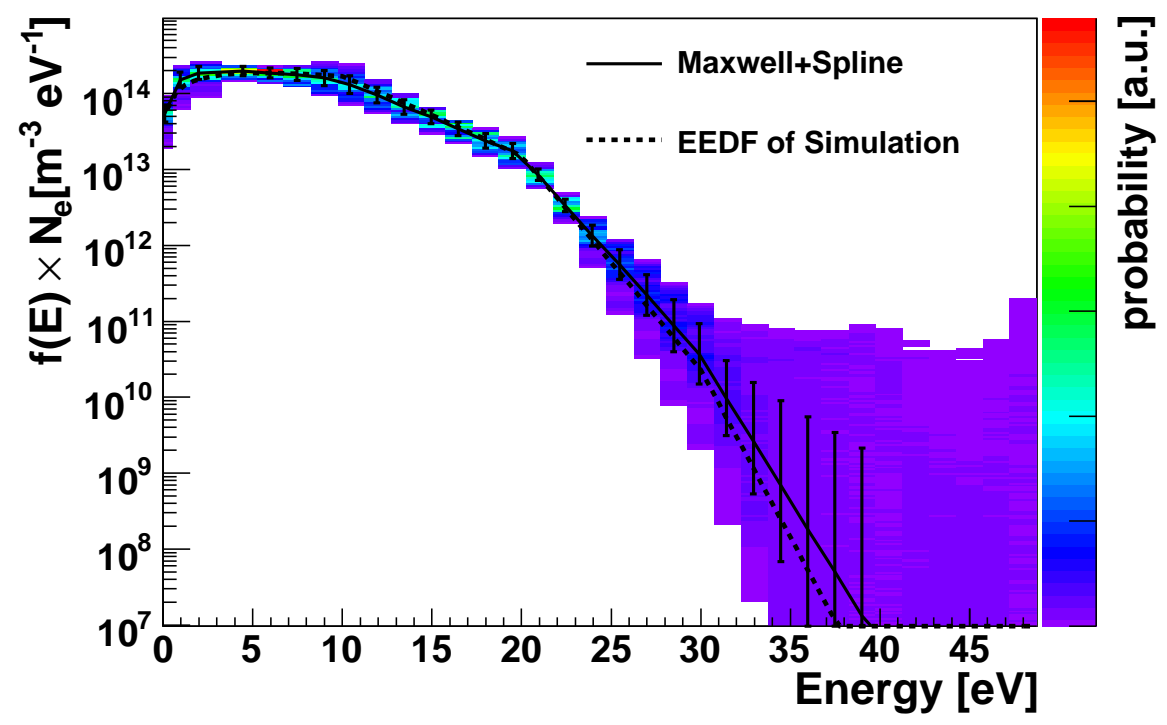

Figure 8. Reconstruction of simulated data. The result of the reconstruction (Maxwell + Spline) is shown together with the EEDF used to simulate the data. The histograms show the marginal posterior distribution for the EEDF at a given energy (see text).

show the marginal distribution of the EEDF at different energies, as sampled during the MC inversion. The histograms are summarized in the overlayed curve with error band, which was obtained by computing the expectation value and variance of the logarithm of the EEDF as a function of energy (equation (19) and equation (20)). The logarithmic scale was chosen, since the marginal distributions show a flat shape in the logarithm of the EEDF, which can be reasonably described by mean and variance in contrast to the strongly asymmetric shape in the linear scale.

For the interpretation of the given confidence region, it is important to keep in mind that the EEDF at adjacent energies is correlated. The correlation length of the EEDF is determined by the flexibility of the employed parameterization. Only a deviation in a region larger than the correlation length indicates a significant disagreement. See also [12] (and references therein) for a discussion of different ways to specify profile uncertainties.

The EEDF is correctly reconstructed in the entire energy range. In the region above $45 \mathrm{eV}$ the error band increases strongly. The EEDF is not constrained by the spectral data in this region. When increasing the flexibility of the parameterization of the EEDF, e.g., by using a higher number of knots of the spline, the error band increases to some extent also in the region between 12 and $19 \mathrm{eV}$. The spectral data only weakly constrains the EEDF in this region. The number of knots of the correction spline was chosen in a way, that the EEDF of kinetic modeling can be correctly described, but no unnecessary flexibility is introduced, which would increase the uncertainty of the reconstruction. 


\subsection{Reconstruction of Measured Data}

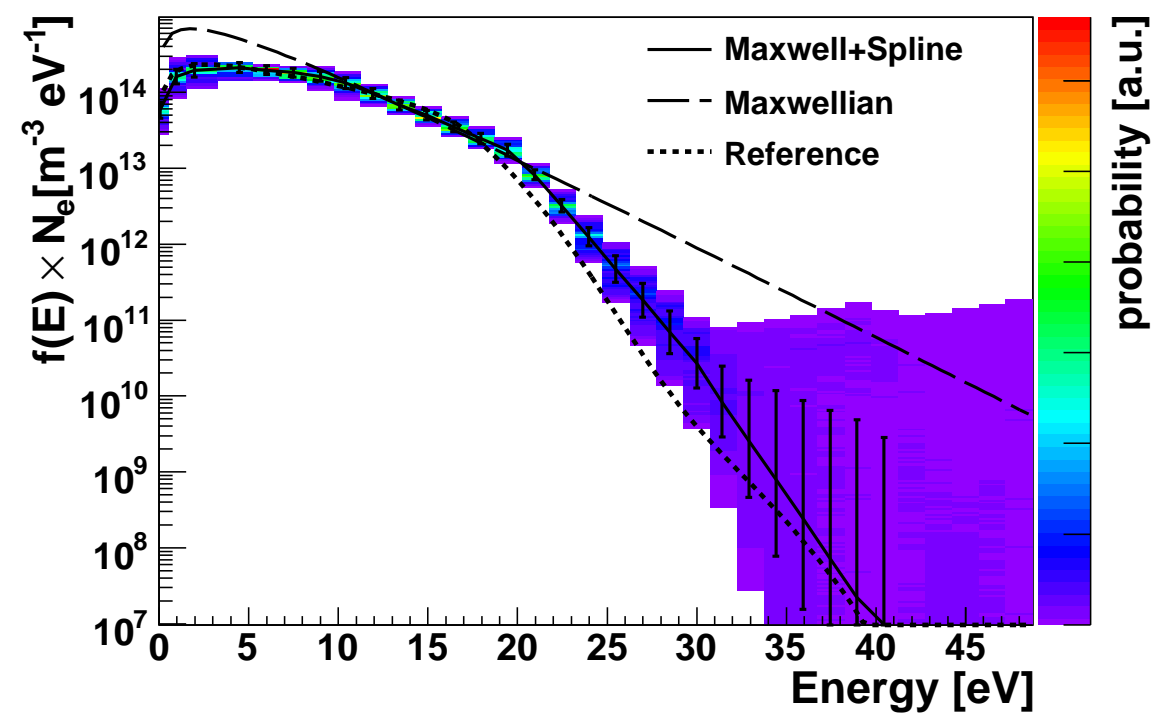

Figure 9. Reconstruction of the EEDF from a neon dc-glow discharge. The histograms show the marginal posterior distribution (color-coded z-axis) of the EEDF at the respective energy, as obtained by Monte-Carlos sampling. The line with error bars summarizes the histograms by showing the expectation value and rms-variance of the logarithm of the EEDF. The Maxwellian with a temperature of $3.5 \mathrm{eV}$ (dashed curve) and the reference distribution obtained by hybrid modeling $([9,22]$, solid curve without error margins) are shown for comparison.

The result of the reconstruction of the EEDF from spectral data measured in the positive column of the discharge is shown in fig. 9. The result of hybrid modeling $[9,22]$ of the EEDF at the center $(r=0)$ of the positive column of an equivalent neon discharge with identical parameters (gas pressure 0.67 Torr, tube radius $1.5 \mathrm{~cm}$, and discharge current $10 \mathrm{~mA}$ ) is shown for comparison. The result of the hybrid model was validated with probe and LIF measurements [9] and acts as reference here. The Maxwellian distribution is shown to demonstrate the deviations from thermal equilibrium, which can be clearly observed.

It can be seen that the distribution is reconstructed up to energies of about $30 \mathrm{eV}$, where it has fallen off to $10^{-4}$ of its maximum value. The shape of the reference distribution is well reproduced, especially in the region below $20 \mathrm{eV}$, where reconstruction and reference agree within the stated uncertainty. Between 20 and $30 \mathrm{eV}$ the reconstructed EEDF slightly exceeds the reference distribution, and the error band is wider in this energy range. Beyond $30 \mathrm{eV}$ the posterior shows a flat shape and only an upper limit for the electron density at these energies can be given.

4.2.1. Energy Dependence of the Elementary Processes. In order to validate the result of the reconstruction with respect to the obtained uncertainty band, the energy 

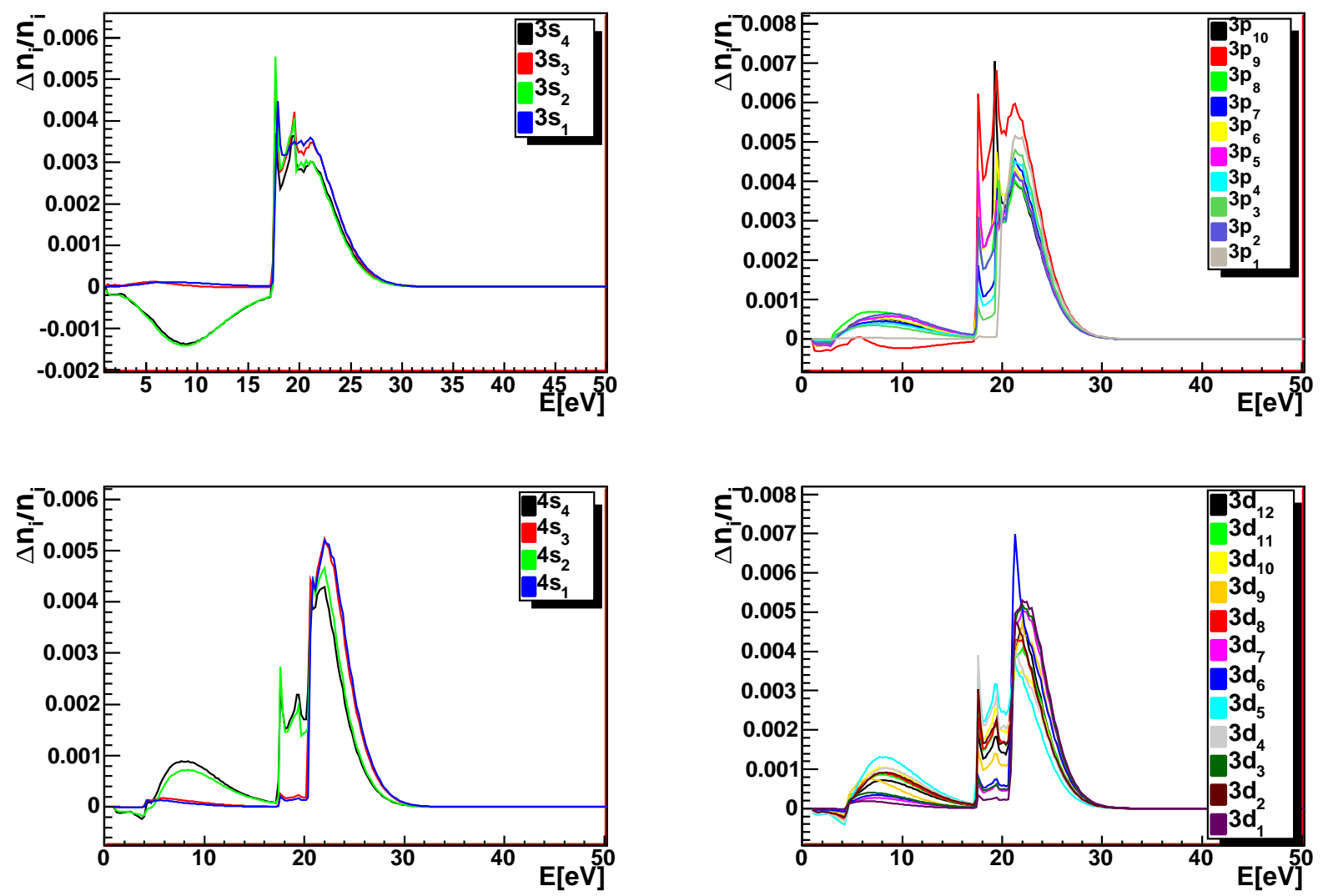

Figure 10. The relative change of the population densities for an increase of the EEDF is shown as function of the energy for the 31 exited states considered in the model. See 3.1 for the notation of the states.

dependence of the elementary processes was considered. The variation of the population densities caused by a change of the EEDF at a given energy was calculated. A piecewise constant parameterization of the EEDF was used:

$$
f_{\mathrm{pw}}(E)=\left\{\begin{array}{ccc}
f_{1} & \text { for } & E<E_{1} \\
f_{2} & \text { for } & E_{1}<E<E_{2} \\
\vdots & & \vdots \\
f_{n} & \text { for } & E_{n-1} E<E_{n}
\end{array}\right.
$$

ach $f_{i}$ was sequentially increased by $10 \%$ and the resulting variations of the population densities $\Delta n_{i} / n_{i}$ were plotted versus the energy $E_{i}$; see fig. 10 . The thresholds for the different excitation and de-excitation channels can be observed clearly (direct excitation, stepwise excitation, and cascade contributions).

The reconstruction of the EEDF is expected to be well determined by the data in energy regions where two conditions are fulfilled: First, the variation of the population densities must not be small and second, there have to be differences in the shape of $\Delta n_{i} / n_{i}(E)$ for some of the excited states. When the values of $\Delta n_{i} / n_{i}(E)$ for all states are proportional to each other, a variation of the EEDF influences all line intensities in 
a similar way. In that case, the EEDF cannot be unambiguously reconstructed.

The uncertainty of the reconstructed EEDF reflects how well these conditions are fulfilled for different energy regions. The uncertainty of the reconstructed EEDF exhibits a minimum around $17 \mathrm{eV}$, where the spread in the variations is large. Above $30 \mathrm{eV}$, where the error band starts to increase considerably, the variation of the population densities also becomes small. In the region between 10 and $17 \mathrm{eV}$, the EEDF is constrained by the chosen parameterization with six spline knots. Samplings with more flexible parameterizations of the EEDF also show an increased error band in this region.

\section{Summary}

A data analysis procedure for the reconstruction of the EEDF from optical emission spectroscopic data was implemented and applied to data from the positive column of a neon dc-discharge. The model of the plasma and the spectroscopic measurement was described by employing a complete and consistent set of atomic structure and electron collision data generated through semi-relativistic $B$-Spline $R$-Matrix (close-coupling) calculations. The probabilistic nature of the approach allowed us to account for the uncertainties of the atomic data set as well as other uncertainties of the model, of experimental or theoretical nature. The consistent propagation of the uncertainties made it possible to state a confidence region of the reconstruction result, which agreed well with published EEDFs from hybrid modeling.

\section{Acknowledgments}

This work was funded by Deutsche Forschungsgemeinschaft through Sonderforschungsbereich Trans Regio 24 (Fundamentals of Complex Plasmas). KB and OZ were supported by the United States National Science Foundation under grants PHY-0757755 and PHY-0555266.

The authors are indebted to V. Dose, H. Dreier, M. Krychowiak, R. Preuss, F. Sigeneger, and D. Uhrlandt for helpful discussions and to H. Testrich and M. Rudolph for help with the production of the discharge tubes.

\section{References}

[1] J. Meichsner: Low Temperature Plasmas. In: Plasma Physics: Confinement, Transport and Collective Effects, Springer Lecture Notes Vol. 670, edited by A. Dinklage et al. Springer, Berlin, (2005)

[2] A. Dinklage, R. Fischer, and J. Svensson: Fusion Sci. and Tech., 46, 355 (2004)

[3] R. Fischer and A. Dinklage: Rev. Sci. Instrum., 75, 4237 (2004)

[4] H. Kang and V. Dose: AIP CP. 707, 295 (2004)

[5] R. Fischer, A. Dinklage, and E. Pasch: Plasma Phys. Control. Fusion, 45, 1095 (2003)

[6] N. Brenning, J. Phys. D: Appl. Phys. 15, L1 (1982)

[7] B. Schweer, G. Mank, A. Pospieszczyk, B. Brosda and B. Pohlmeyer: J. Nucl. Mat. 196-198 (1992) 174-178 
[8] R. Fischer and V. Dose, Plasma Phys. Control. Fusion 41, 1109 (1999)

[9] D. Uhrlandt and S. Franke, J. Phys. D: Appl. Phys. 35, 680 (2002)

[10] D. Loffhagen, F. Sigeneger and R. Winkler, J. Phys. D: Appl. Phys. 35, 1768 (2002)

[11] A. Beck, D. Hemmers, H. Kempkens, H. B. Schweer and J. Uhlenbusch, J. Phys. D: Appl. Phys. 33, $360(2000)$

[12] R.Fischer, E. Wolfrum, J. Schweinzer, and the ASDEX Upgrade Team Probabilistic Lithium Beam Data Analysis Plasma Phys. Control. Fusion, 50, 1-26 (2008)

[13] Dirk Dodt, Andreas Dinklage, Rainer Fischer, and Detlef Loffhagen, AIP Conf. Proc., 872, 264 (2006), Dirk Dodt, Andreas Dinklage, Rainer Fischer, and Roland Preuss, AIP Conf. Proc. 954, 468 (2007), Dirk Dodt, Andreas Dinklage, Rainer Fischer, Klaus Bartschat, and Oleg Zatsarinny, AIP Conf. Proc. 993, 203 (2007), NIFS Proc. 69, 841 (2007)

[14] R. Preuss, A. Dinklage and A. Weller, Phys. Rev. Lett. 99245001 (2007).

[15] Markov Chain Monte Carlo in Practice, edited by W.R. Gilks, S. Richardson, and D.J. Spiegelhalter, Chapmann \& Hall, Boca Raton, Florida (1996)

[16] J. E. Lawler and J.J. Curry, J. Phys. D: Appl. Phys. 31, 3235 (1998)

[17] L. W. G. Steenhuijsen, Contrib. Plasma Phys. 21, 301 (1981)

[18] R. Wetzel, F. Baiocchi, T. Hayes, and R. Freund, Phys. Rev. A 35, 559 (1987)

[19] L. Vriens and A. H. M. Smeets, Phys. Rev. A 22, 940 (1980)

[20] P. Jonas, B. Bruhn, B.-P. Koch, and A. Dinklage, Phys. Plasmas 7729 (2000), with Erratum Phys. Plasmas 73481 (2000)

[21] K. Wiesemann Einführung in die Gaselektronik, Teubner Studienbücher Physik, Stuttgard (1976)

[22] D. Uhrlandt, INP Greifswald, private Communications. (2006)

[23] D. F. Gray The Observation and Analysis of Stellar Photospheres, Second edition, Cambridge University Press, Bristol (1992)

[24] V. Dose, R. Fischer, and W. von der Linden, Maximum Entropy and Bayesian Methods, edited by G. Erickson, Kluwer Academic, Dordrecht (1998).

[25] P. Gregory Bayesian Logical Data Analysis for the Physical Sciences (Cambridge University Press, Cambridge, 2005)

[26] O. Zatsarinny and K. Bartschat, J. Phys. B: At. Mol. Opt. Phys. 37, 2173 (2004)

[27] M. Allan, K. Franz, H. Hotop, O. Zatsarinny, and K. Bartschat, J. Phys. B: At. Mol. Opt. Phys. 39, L139 (2006)

[28] Yu Ralchenko, F.-C. Jou, D.E. Kelleher, A.E. Kramida, A. Musgrove, J. Reader, W.L. Wiese, and K. Olsen (2007). NIST Atomic Spectra Database (version 3.1.3), [Online]. Available: http://physics.nist.gov/asd3 [2007, October 11]. National Institute of Standards and Technology, Gaithersburg, MD, USA.

[29] M. Allan, K. Franz, H. Hotop, O. Zatsarinny, and K. Bartschat, J. Phys. B: At. Mol. Opt. Phys. 39, L139 (2006)

[30] Crow EL, Shimizu K Log-normal Distributions: Theory and Application Dekker, New York (1988)

[31] C. Ballance and D. Griffin, J. Phys. B: At. Mol. Opt. Phys. 37 2943-2957 (2004)

[32] J. Chilton, M. Stewart, and Chun C. Lin, Phys. Rev. A 61052708 (2000)

[33] K. A. Berrington, W. Eissner, P.H. Norrington, Comput. Phys. Commun. 92, 290, (1995)

[34] D.S. Sivia: Data Analysis: A Bayesian Tutorial, 2nd edition, Oxford University Press, Oxford (2006)

[35] A. Werner, IPP Greifswald, private Communications. (2006)- 